\title{
Generalized fractional Grüss-type inequalities
}

\author{
Paulo M. Guzmán ${ }^{1,2,3}$, Juan E. Nápoles Valdés ${ }^{1,4, *}$ \\ ${ }^{1}$ Facultad de Ciencias Exactas y Naturales y Agrimensura, Universidad Nacional del Nordeste, Av. Libertad 5450, Corrientes, Argentina \\ ${ }^{2}$ Facultad de Ciencias Agrarias, Universidad Nacional del Nordeste, Sargento Cabral 2131, Corrientes, Argentina \\ ${ }^{3}$ Facultad de Ingeniería, Universidad Nacional del Nordeste, Av. Las Heras 727, Resistencia, Chaco, Argentina \\ ${ }^{4}$ Facultad Regional Resistencia, Universidad Tecnologica Nacional, French 414, Resistencia, Chaco, Argentina
}

(Received: 5 October 2020. Received in revised form: 3 November 2020. Accepted: 6 November 2020. Published online: 13 November 2020.)

(C) 2020 the authors. This is an open access article under the CC BY (International 4.0) license (www.creativecommons.org/licenses/by/4.0/).

\begin{abstract}
In this paper, using a generalized integral operator of the Riemann-Liouville type defined in a previous paper, we present several integral inequalities of Grüss type. Several existing results are obtained as the special cases of the results derived in this paper.
\end{abstract}

Keywords: Grüss inequality; generalized fractional integral.

2020 Mathematics Subject Classification: 26A33, 26D10, 26D15.

\section{Introduction}

The following result (Theorem 1.1) contains an interesting and useful integral inequality that gives an estimate of the difference between the integral of the product of two functions and the product of their integrals. This inequality is known as the Grüss inequality $[9,18,19]$.

Theorem 1.1. Let $a$ and $b$ be two real numbers satisfying the inequality $a<b$, and let $\varphi, \psi:[a, b] \rightarrow \mathbb{R}$ be two integrable functions such that $m \leq f(\chi) \leq M$ and $k \leq g(\chi) \leq K$ for all $\chi \in[a, b]$, where $m, M, k$ and $K$ are constants. Then, the following inequality holds

$$
\left|\frac{1}{b-a} \int_{a}^{b} \varphi(\chi) g(\chi) d \chi-\frac{1}{b-a} \int_{a}^{b} \varphi(\chi) d \chi \frac{1}{b-a} \int_{a}^{b} g(\chi) d \chi\right| \leq \frac{1}{4}(M-m)(K-k),
$$

where the constant $\frac{1}{4}$ is sharp.

Grüss-type inequalities have found various applications in different areas of mathematics, including difference equations. Such inequalities have also been used to estimate and extend the Mellin transform in different contexts. In recent years, inequality (1) has been the object of attention from many researchers and consequently numerous results have been obtained in various contexts; for example, see [2-5, 7, 8, 14, 15, 17, 20,21] and the references cited therein.

The emergence of fractional calculus has given rise to an area that has a lot of interaction with different mathematical and scientific topics. Much development in this area has already been made. In this development, different types of integral and differential fractional operators have been emerged, including Riemann-Liouville, Caputo-Fabrizio and Katugampola. In the literature, there are various types of integrals with singular and non-singular kernels.

In [10], we defined the generalized integral operator given in Definition 1.1, studied its fundamental properties and applied it to study the various Hermite-Hadamard inequalities.

Definition 1.1. The generalized fractional Riemann-Liouville integral of order $\alpha$ of an integrable function $\varphi(\chi)$ on $[0, \infty)$, is given as follows:

$$
\left({ }^{\beta} J_{F, a+}^{\alpha} \varphi\right)(\chi)=\frac{1}{\Gamma(\beta)} \int_{a}^{\chi} \frac{\varphi(\tau) d \tau}{F(\mathbb{F}(\chi, \tau), \beta) F(\tau, \alpha)},
$$

where $\alpha \in \mathbb{R}, F$ is an absolutely continuous positive function and $\mathbb{F}(\chi, \tau)=\int_{\tau}^{\chi} \frac{d s}{F(\tau, s)}$.

Under the conditions of Definition 1.1, we enunciate the left and right integral operators given in the next definition.

*Corresponding author (jnapoles@exa.unne.edu.ar) 
Definition 1.2. The left and right fractional generalized integrals of order $\beta \in \mathbb{C}, \operatorname{Re}(\beta)>0$, are defined by

$$
\begin{aligned}
\left({ }^{\beta} J_{F, a+}^{\alpha} \varphi\right)(\chi) & =\frac{1}{\Gamma(\beta)} \int_{a}^{\chi} \frac{\varphi(\tau) d \tau}{F\left(\mathbb{F}_{+}(\chi, \tau), \beta\right) F(\tau-a, \alpha)}, \\
\left({ }^{\beta} J_{F, b-}^{\alpha} \varphi\right)(\chi) & =\frac{1}{\Gamma(\beta)} \int_{\chi}^{b} \frac{\varphi(\tau) d \tau}{F\left(\mathbb{F}_{-}(\tau, \chi), \beta\right) F(b-\tau, \alpha)}
\end{aligned}
$$

where

$$
\begin{aligned}
\mathbb{F}_{+}(\chi, \tau) & =\int_{\tau}^{\chi} \frac{d s}{F(s-a, \alpha)}, \\
\mathbb{F}_{-}(\tau, \chi) & =\int_{\chi}^{\tau} \frac{d s}{F(b-s, \alpha)}
\end{aligned}
$$

and $F\left(\mathbb{F}_{+}(\tau, \chi), 1\right)=F\left(\mathbb{F}_{-}(\tau, \chi), 1\right)=1$.

In this article, our purpose is to establish various integral inequalities of the Grüss type, in the context of the generalized integral operators mentioned in Definition 1.2.

\section{Main results}

In this section, we obtain certain integral inequalities of the Grüss type, in the context of the integral operators given in Definition 1.2.

Theorem 2.1. Consider an integrable function $\varphi$ defined on $[0, \infty)$, and suppose there are two integrable functions $\lambda_{1}$ and $\lambda_{2}$ on $[0, \infty)$ satisfying

$$
\lambda_{1}(\tau) \leq \varphi(\tau) \leq \lambda_{2}(\tau), \quad \forall \tau \in[0, \infty) .
$$

Then, the following inequality for generalized fractional integrals holds

$$
\left({ }^{\beta} J_{F, 0+}^{\alpha} \lambda_{2}\right)(\tau)\left({ }^{\gamma} J_{F, 0+}^{\delta} \varphi\right)(\tau)+\left({ }^{\beta} J_{F, 0+}^{\alpha} \varphi\right)(\tau)\left({ }^{\gamma} J_{F, 0+}^{\delta} \lambda_{1}\right)(\tau) \geq\left({ }^{\beta} J_{F, 0+}^{\alpha} \lambda_{2}\right)(\tau)\left({ }^{\gamma} J_{F, 0+}^{\delta} \lambda_{1}\right)(\tau)+\left({ }^{\beta} J_{F, 0+}^{\alpha} \varphi\right)(\tau)\left({ }^{\gamma} J_{F, 0+}^{\delta} \varphi\right)(\tau)
$$

for all $\tau>0, \alpha, \delta \in \mathbb{R}$ and $\beta, \gamma \in \mathbb{C}$ with $\operatorname{Re}(\beta)>0$ and $\operatorname{Re}(\gamma)>0$.

Proof. From (4), it follows that

$$
\left[\lambda_{2}(\chi)-\varphi(\chi)\right]\left[\varphi(\xi)-\lambda_{1}(\xi)\right] \geq 0
$$

for all $\chi \geq 0$ and $\xi \geq 0$. Hence,

$$
\lambda_{2}(\chi) \varphi(\xi)+\lambda_{1}(\chi) \varphi(\chi) \geq \lambda_{1}(\xi) \lambda_{2}(\chi)+\varphi(\chi) \varphi(\xi) .
$$

Multiply (6) with $\frac{1}{\Gamma(\beta)} \frac{1}{F\left(\mathbb{F}_{+}(\chi, \tau), \beta\right) F(\tau, \alpha)}$ and integrating the resulting inequality with respect to $\chi$ on $(0, \tau)$, we get

$$
\begin{aligned}
& \frac{\varphi(\xi)}{\Gamma(\beta)} \int_{0}^{\tau} \frac{\lambda_{2}(\chi) d \chi}{F\left(\mathbb{F}_{+}(\chi, \tau), \beta\right) F(\tau, \alpha)}+\frac{\lambda_{1}(\xi)}{\Gamma(\beta)} \int_{0}^{\tau} \frac{\varphi(\chi) d \chi}{F\left(\mathbb{F}_{+}(\chi, \tau), \beta\right) F(\tau, \alpha)} \\
\geq & \frac{\lambda_{1}(\xi)}{\Gamma(\beta)} \int_{0}^{\tau} \frac{\lambda_{2}(\chi) d \chi}{F\left(\mathbb{F}_{+}(\chi, \tau), \beta\right) F(\tau, \alpha)}+\frac{\varphi(\xi)}{\Gamma(\beta)} \int_{0}^{\tau} \frac{\varphi(\chi) d \chi}{F\left(\mathbb{F}_{+}(\chi, \tau), \beta\right) F(\tau, \alpha)} .
\end{aligned}
$$

Using (2), we can write this inequality as

$$
\varphi(\xi)\left({ }^{\beta} J_{F, 0+}^{\alpha} \lambda_{2}\right)(\tau)+\lambda_{1}(\xi)\left({ }^{\beta} J_{F, 0+}^{\alpha} \varphi\right)(\tau) \geq \lambda_{1}(\xi)\left({ }^{\beta} J_{F, 0+}^{\alpha} \lambda_{2}\right)(\tau)+\varphi(\xi)\left({ }^{\beta} J_{F, 0+}^{\alpha} \varphi\right)(\tau) .
$$

To obtain the desired result, we multiply (7) with $\frac{1}{\Gamma(\gamma)} \frac{1}{F\left(\mathbb{F}_{+}(\xi, \tau), \gamma\right) F(\tau, \delta)}$ and integrate the resulting inequality with respect to $\xi$ on $(0, \tau)$ :

$$
\begin{aligned}
& \left({ }^{\beta} J_{F, 0+}^{\alpha} \lambda_{2}\right)(\tau) \frac{1}{\Gamma(\gamma)} \int_{0}^{\tau} \frac{\varphi(\xi) d \xi}{F\left(\mathbb{F}_{+}(\xi, \tau), \gamma\right) F(\tau, \delta)}+\left({ }^{\beta} J_{F, 0+}^{\alpha} \varphi\right)(\tau) \frac{1}{\Gamma(\gamma)} \int_{0}^{\tau} \frac{\lambda_{1}(\xi) d \xi}{F\left(\mathbb{F}_{+}(\xi, \tau), \gamma\right) F(\tau, \delta)} \\
\geq & \left({ }^{\beta} J_{F, 0+}^{\alpha} \lambda_{2}\right)(\tau) \frac{1}{\Gamma(\gamma)} \int_{0}^{\tau} \frac{\lambda_{1}(\xi) d \xi}{F\left(\mathbb{F}_{+}(\xi, \tau), \gamma\right) F(\tau, \delta)}+\left({ }^{\beta} J_{F, 0+}^{\alpha} \varphi\right)(\tau) \frac{1}{\Gamma(\gamma)} \int_{0}^{\tau} \frac{\varphi(\xi) d \xi}{F\left(\mathbb{F}_{+}(\xi, \tau), \gamma\right) F(\tau, \delta)},
\end{aligned}
$$

which can be rewritten, by using (2), as follows

$$
\left({ }^{\beta} J_{F, 0+}^{\alpha} \lambda_{2}\right)(\tau)\left({ }^{\gamma} J_{F, 0+}^{\delta} \varphi\right)(\tau)+\left({ }^{\beta} J_{F, 0+}^{\alpha} \varphi\right)(\tau)\left({ }^{\gamma} J_{F, 0+}^{\delta} \lambda_{1}\right)(\tau) \geq\left({ }^{\beta} J_{F, 0+}^{\alpha} \lambda_{2}\right)(\tau)\left({ }^{\gamma} J_{F, 0+}^{\delta} \lambda_{1}\right)(\tau)+\left({ }^{\beta} J_{F, 0+}^{\alpha} \varphi\right)(\tau)\left({ }^{\gamma} J_{F, 0+}^{\delta} \varphi\right)(\tau),
$$

that is the required inequality. 
Remark 2.1. As a particular case, if we take $F(\tau, \alpha)=\tau^{1-\alpha}$ then Theorem 2.1 is same as Theorem 2.1 of [4].

Remark 2.2. Under the condition of Remark 2.1, if additionally we take $\beta=1$ then Theorem 2.1 becomes Theorem 2 of [23].

The following result incorporates a function $\psi(\tau)$, which leads us to a double version of Grüss's inequality.

Theorem 2.2. If in addition to the considerations of Theorem 2.1, we assume that there is an integrable function $\psi$ and two integrable functions $\eta_{1}, \eta_{2}$ on $[0, \infty)$ satisfying

$$
\eta_{1}(\tau) \leq \psi(\tau) \leq \eta_{2}(\tau), \quad \forall \tau \in[0, \infty),
$$

then the following inequalities for generalized fractional integrals hold

$$
\begin{aligned}
& \left({ }^{\beta} J_{F, 0+}^{\alpha} \lambda_{2}\right)(\tau)\left({ }^{\gamma} J_{F, 0+}^{\delta} \psi\right)(\tau)+\left({ }^{\beta} J_{F, 0+}^{\alpha} \varphi\right)(\tau)\left({ }^{\gamma} J_{F, 0+}^{\delta} \eta_{1}\right)(\tau) \geq\left({ }^{\beta} J_{F, 0+}^{\alpha} \lambda_{2}\right)(\tau)\left({ }^{\gamma} J_{F, 0+}^{\delta} \eta_{1}\right)(\tau)+\left({ }^{\beta} J_{F, 0+}^{\alpha} \varphi\right)(\tau)\left({ }^{\gamma} J_{F, 0+}^{\delta} \psi\right)(\tau),(9) \\
& \left({ }^{\beta} J_{F, 0+}^{\alpha} \eta_{2}\right)(\tau)\left({ }^{\gamma} J_{F, 0+}^{\delta} \varphi\right)(\tau)+\left({ }^{\beta} J_{F, 0+}^{\alpha} \psi\right)(\tau)\left({ }^{\gamma} J_{F, 0+}^{\delta} \lambda_{1}\right)(\tau) \geq\left({ }^{\beta} J_{F, 0+}^{\alpha} \eta_{2}\right)(\tau)\left({ }^{\gamma} J_{F, 0+}^{\delta} \lambda_{1}\right)(\tau)+\left({ }^{\beta} J_{F, 0+}^{\alpha} \psi\right)(\tau)\left({ }^{\gamma} J_{F, 0+}^{\delta} \varphi\right)(\tau), \\
& (10) \\
& \left({ }^{\beta} J_{F, 0+}^{\alpha} \lambda_{2}\right)(\tau)\left({ }^{\gamma} J_{F, 0+}^{\delta} \eta_{2}\right)(\tau)+\left({ }^{\beta} J_{F, 0+}^{\alpha} \varphi\right)(\tau)\left({ }^{\gamma} J_{F, 0+}^{\delta} \psi\right)(\tau) \geq\left({ }^{\beta} J_{F, 0+}^{\alpha} \lambda_{2}\right)(\tau)\left({ }^{\gamma} J_{F, 0+}^{\delta} \psi\right)(\tau)+\left({ }^{\gamma} J_{F, 0+}^{\delta} \eta_{2}\right)(\tau)\left({ }^{\beta} J_{F, 0+}^{\alpha} \varphi\right)(\tau), \\
& (11) \\
& \left({ }^{\beta} J_{F, 0+}^{\alpha} \lambda_{1}\right)(\tau)\left({ }^{\gamma} J_{F, 0+}^{\delta} \eta_{1}\right)(\tau)+\left({ }^{\beta} J_{F, 0+}^{\alpha} \varphi\right)(\tau)\left({ }^{\gamma} J_{F, 0+}^{\delta} \psi\right)(\tau) \geq\left({ }^{\beta} J_{F, 0+}^{\alpha} \lambda_{1}\right)(\tau)\left({ }^{\gamma} J_{F, 0+}^{\delta} \psi\right)(\tau)+\left({ }^{\gamma} J_{F, 0+}^{\delta} \eta_{1}\right)(\tau)\left({ }^{\beta} J_{F, 0+}^{\alpha} \varphi\right)(\tau),
\end{aligned}
$$

for all $\tau>0, \alpha, \delta \in \mathbb{R}$ and $\beta, \gamma \in \mathbb{C}$ with $\operatorname{Re}(\beta)>0$ and $\operatorname{Re}(\gamma)>0$.

Proof. We firstly prove (9). Using relations (4) and (8), we have

$$
\left[\lambda_{2}(\chi)-\varphi(\chi)\right]\left[\psi(\xi)-\eta_{1}(\xi)\right] \geq 0,
$$

for $\tau \in[0, \infty)$. Hence,

$$
\lambda_{2}(\chi) \psi(\xi)+\eta_{1}(\xi) \varphi(\chi) \geq \eta_{1}(\xi) \lambda_{2}(\chi)+\varphi(\chi) \psi(\xi) .
$$

Multiplying both sides of (13) with $\frac{1}{\Gamma(\beta)} \frac{1}{F\left(\mathbb{F}_{+}(\chi, \tau), \beta\right) F(\tau, \alpha)}$ and integrating the resulting inequality with respect to $\chi$ on $(0, \tau)$, we obtain

$$
\begin{aligned}
& \frac{\psi(\xi)}{\Gamma(\beta)} \int_{0}^{\tau} \frac{\lambda_{2}(\chi) d \chi}{F\left(\mathbb{F}_{+}(\chi, \tau), \beta\right) F(\tau, \alpha)}+\frac{\eta_{1}(\xi)}{\Gamma(\beta)} \int_{0}^{\tau} \frac{\varphi(\chi) d \chi}{F\left(\mathbb{F}_{+}(\chi, \tau), \beta\right) F(\tau, \alpha)} \\
\geq & \frac{\eta_{1}(\xi)}{\Gamma(\beta)} \int_{0}^{\tau} \frac{\lambda_{2}(\chi) d \chi}{F\left(\mathbb{F}_{+}(\chi, \tau), \beta\right) F(\tau, \alpha)}+\frac{\psi(\xi)}{\Gamma(\beta)} \int_{0}^{\tau} \frac{\varphi(\chi) d \chi}{F\left(\mathbb{F}_{+}(\chi, \tau), \beta\right) F(\tau, \alpha)} .
\end{aligned}
$$

By using Definition 1.2, we have

$$
\psi(\xi)\left({ }^{\beta} J_{F, 0+}^{\alpha} \lambda_{2}\right)(\tau)+\eta_{1}(\xi)\left({ }^{\beta} J_{F, 0+}^{\alpha} \varphi\right)(\tau) \geq \eta_{1}(\xi)\left({ }^{\beta} J_{F, 0+}^{\alpha} \lambda_{2}\right)(\tau)+\psi(\xi)\left({ }^{\beta} J_{F, 0+}^{\alpha} \varphi\right)(\tau) .
$$

Multiplying this last inequality by $\frac{1}{\Gamma(\gamma)} \frac{1}{F\left(\mathbb{F}_{+}(\xi, \tau), \gamma\right) F(\tau, \delta)}$ and integrating the resulting inequality with respect to $\xi$ on $(0, \tau)$, we get

$$
\begin{aligned}
& \left({ }^{\beta} J_{F, 0+}^{\alpha} \lambda_{2}\right)(\tau) \frac{1}{\Gamma(\gamma)} \int_{0}^{\tau} \frac{\psi(\xi) d \xi}{F\left(\mathbb{F}_{+}(\xi, \tau), \gamma\right) F(\tau, \delta)}+\left({ }^{\beta} J_{F, 0+}^{\alpha} \varphi\right)(\tau) \frac{1}{\Gamma(\gamma)} \int_{0}^{\tau} \frac{\eta_{1}(\xi) d \xi}{F\left(\mathbb{F}_{+}(\xi, \tau), \gamma\right) F(\tau, \delta)} \\
\geq & \left({ }^{\beta} J_{F, 0+}^{\alpha} \lambda_{2}\right)(\tau) \frac{1}{\Gamma(\gamma)} \int_{0}^{\tau} \frac{\eta_{1}(\xi) d \xi}{F\left(\mathbb{F}_{+}(\xi, \tau), \gamma\right) F(\tau, \delta)}+\left({ }^{\beta} J_{F, 0+}^{\alpha} \varphi\right)(\tau) \frac{1}{\Gamma(\gamma)} \int_{0}^{\tau} \frac{\psi(\xi) d \xi}{F\left(\mathbb{F}_{+}(\xi, \tau), \gamma\right) F(\tau, \delta)} .
\end{aligned}
$$

This inequality is equivalent to

$$
\left({ }^{\beta} J_{F, 0+}^{\alpha} \lambda_{2}\right)(\tau)\left({ }^{\gamma} J_{F, 0+}^{\delta} \psi\right)(\tau)+\left({ }^{\beta} J_{F, 0+}^{\alpha} \varphi\right)(\tau)\left({ }^{\gamma} J_{F, 0+}^{\delta} \eta_{1}\right)(\tau) \geq\left({ }^{\beta} J_{F, 0+}^{\alpha} \lambda_{2}\right)(\tau)\left({ }^{\gamma} J_{F, 0+}^{\delta} \eta_{1}\right)(\tau)+\left({ }^{\beta} J_{F, 0+}^{\alpha} \varphi\right)(\tau)\left({ }^{\gamma} J_{F, 0+}^{\delta} \psi\right)(\tau)
$$

which brings us to the required inequality (9).

To get (10)-(12), it is enough to use the inequalities

$$
\begin{aligned}
& {\left[\eta_{2}(\chi)-\psi(\chi)\right]\left[\varphi(\xi)-\lambda_{1}(\xi)\right] \geq 0} \\
& {\left[\lambda_{2}(\chi)-\varphi(\chi)\right]\left[\psi(\xi)-\eta_{2}(\xi)\right] \leq 0} \\
& {\left[\lambda_{1}(\chi)-\varphi(\chi)\right]\left[\psi(\xi)-\eta_{1}(\xi)\right] \leq 0}
\end{aligned}
$$

respectively, and proceed as in the proof of (9). 
Remark 2.3. If we take $F(\tau, \alpha)=\tau^{1-\alpha}$ then Theorem 2.2 coincides with Theorem 2.2 of [4].

Remark 2.4. If we also take $\beta=1$ then then Theorem 2.2 gives Theorem 5 of [23].

Lemma 2.1. Let $\varphi, \lambda_{1}$ and $\lambda_{2}$ be integrable functions on $[0, \infty)$. Suppose the condition (4) is satisfied. For $\tau>0$, $\alpha \in \mathbb{R}$ and $\beta \in \mathbb{C}$ with $\operatorname{Re}(\beta)>0$, the following equality holds for the generalized fractional integral operator:

$$
\begin{aligned}
& {\left[\left({ }^{\beta} J_{0+}^{\alpha} \lambda_{2}\right)(\tau)-\left({ }^{\beta} J_{0+}^{\alpha} \varphi\right)(\tau)\right]\left[\left({ }^{\beta} J_{0+}^{\alpha} \varphi\right)(\tau)-\left({ }^{\beta} J_{0+}^{\alpha} \lambda_{1}\right)(\tau)\right]-\left[{ }^{\beta} J_{0+}^{\alpha}\left(\left(\lambda_{2}(\tau)-\varphi(\tau)\right)\left(\varphi(\tau)-\lambda_{1}(\tau)\right)\right)\right]\left(1+{ }^{\beta} J_{0+}^{\alpha}(1)\right) } \\
= & \left({ }^{\beta} J_{0+}^{\alpha} \varphi^{2}\right)(\tau)^{\beta} J_{0+}^{\alpha}(1)-\left(\left({ }^{\beta} J_{0+}^{\alpha} \varphi\right)(\tau)\right)^{2}+\left({ }^{\beta} J_{0+}^{\alpha} \lambda_{2}\right)(\tau)\left({ }^{\beta} J_{0+}^{\alpha} \varphi\right)(\tau)+\left({ }^{\beta} J_{0+}^{\alpha} \lambda_{1}\right)(\tau)\left({ }^{\beta} J_{0+}^{\alpha} \varphi\right)(\tau) \\
& -\left({ }^{\beta} J_{0+}^{\alpha} \lambda_{2}\right)(\tau)\left({ }^{\beta} J_{0+}^{\alpha} \lambda_{1}\right)(\tau)-\left({ }^{\beta} J_{0+}^{\alpha} \lambda_{2} \varphi\right)(\tau)^{\beta} J_{0+}^{\alpha}(1)+\left({ }^{\beta} J_{0+}^{\alpha} \lambda_{1} \lambda_{2}\right)(\tau)^{\beta} J_{0+}^{\alpha}(1)-\left({ }^{\beta} J_{0+}^{\alpha} \lambda_{1} \varphi\right)(\tau)^{\beta} J_{0+}^{\alpha}(1) .
\end{aligned}
$$

Proof. For any $\chi>0$ and $\xi>0$, the following inequality is valid

$$
\begin{aligned}
& {\left[\lambda_{2}(\xi)-\varphi(\xi)\right]\left[\varphi(\chi)-\lambda_{1}(\chi)\right]+\left[\lambda_{2}(\chi)-\varphi(\chi)\right]\left[\varphi(\xi)-\lambda_{1}(\xi)\right]-\left[\lambda_{2}(\chi)-\varphi(\chi)\right]\left[\varphi(\chi)-\lambda_{1}(\chi)\right]-\left[\lambda_{2}(\xi)-\varphi(\xi)\right]\left[\varphi(\xi)-\lambda_{1}(\xi)\right]} \\
& =\varphi^{2}(\chi)+\varphi^{2}(\xi)-2 \varphi(\chi) \varphi(\xi)+\lambda_{2}(\xi) \varphi(\chi)+\lambda_{1}(\chi) \varphi(\xi)-\lambda_{1}(\chi) \lambda_{2}(\xi) \\
& +\lambda_{2}(\chi) \varphi(\xi)+\lambda_{1}(\xi) \varphi(\chi)-\lambda_{1}(\xi) \lambda_{2}(\chi)-\lambda_{2}(\chi) \varphi(\chi)+\lambda_{1}(\chi) \lambda_{2}(\chi)-\lambda_{1}(\chi) \varphi(\chi)-\lambda_{2}(\xi) \varphi(\xi)+\lambda_{1}(\xi) \lambda_{2}(\xi)-\lambda_{1}(\xi) \varphi(\xi) .
\end{aligned}
$$

Multiplying this inequality by $\frac{1}{\Gamma(\beta)} \frac{1}{F\left(\mathbb{F}_{+}(\chi, \tau), \beta\right) F(\tau, \alpha)}$ and integrating the result obtained, with respect to $\chi$ from 0 to $\tau$, we obtain

$$
\begin{aligned}
& {\left[\lambda_{2}(\xi)-\varphi(\xi)\right]\left[\left({ }^{\beta} J_{0+}^{\alpha} \varphi\right)(\tau)-\left({ }^{\beta} J_{0+}^{\alpha} \lambda_{1}\right)(\tau)\right]+\left[\left({ }^{\beta} J_{0+}^{\alpha} \lambda_{2}\right)(\tau)-\left({ }^{\beta} J_{0+}^{\alpha} \varphi\right)(\tau)\right]\left[\varphi(\xi)-\lambda_{1}(\xi)\right]} \\
& -\left[{ }^{\beta} J_{0+}^{\alpha}\left(\left(\lambda_{2}(\tau)-\varphi(\tau)\right)\left(\varphi(\tau)-\lambda_{1}(\tau)\right)\right)\right]-\left(\lambda_{2}(\xi)-\varphi(\xi)\right)\left(\varphi(\xi)-\lambda_{1}(\xi)\right)^{\beta} J_{0+}^{\alpha}(1) \\
& =\left({ }^{\beta} J_{0+}^{\alpha} \varphi^{2}\right)(\tau)+\varphi^{2}(\xi)^{\beta} J_{0+}^{\alpha}(1)-2 \varphi(\xi)\left({ }^{\beta} J_{0+}^{\alpha} \varphi\right)(\tau)+\lambda_{2}(\xi)\left({ }^{\beta} J_{0+}^{\alpha} \varphi\right)(\tau)+\varphi(\xi)\left({ }^{\beta} J_{0+}^{\alpha} \lambda_{1}\right)(\tau)-\lambda_{2}(\xi)\left({ }^{\beta} J_{0+}^{\alpha} \lambda_{1}\right)(\tau) \\
& \quad+\varphi(\xi)\left({ }^{\beta} J_{0+}^{\alpha} \lambda_{2}\right)(\tau)+\lambda_{1}(\xi)\left({ }^{\beta} J_{0+}^{\alpha} \varphi\right)(\tau)-\lambda_{1}(\xi)\left({ }^{\beta} J_{0+}^{\alpha} \lambda_{2}\right)(\tau)-\left({ }^{\beta} J_{0+}^{\alpha} \lambda_{2} \varphi\right)(\tau)+\left({ }^{\beta} J_{0+}^{\alpha} \lambda_{1} \lambda_{2}\right)(\tau) \\
& -\left({ }^{\beta} J_{0+}^{\alpha} \lambda_{1} \varphi\right)(\tau)-\lambda_{2}(\xi) \varphi(\xi)^{\beta} J_{0+}^{\alpha}(1)+\lambda_{1}(\xi) \lambda_{2}(\xi)^{\beta} J_{0+}^{\alpha}(1)-\lambda_{1}(\xi) \varphi(\xi)^{\beta} J_{0+}^{\alpha}(1) .
\end{aligned}
$$

Now, we multiply the above inequality by $\frac{1}{\Gamma(\beta)} \frac{1}{F\left(\mathbb{F}_{+}(\xi, \tau), \beta\right) F(\tau, \alpha)}$ and integrating the resulting inequality with respect to $\xi$, from 0 to $\tau$, we get

$$
\begin{aligned}
& {\left[\left({ }^{\beta} J_{0+}^{\alpha} \lambda_{2}\right)(\tau)-\left({ }^{\beta} J_{0+}^{\alpha} \varphi\right)(\tau)\right]\left[\left({ }^{\beta} J_{0+}^{\alpha} \varphi\right)(\tau)-\left({ }^{\beta} J_{0+}^{\alpha} \lambda_{1}\right)(\tau)\right]+\left[\left({ }^{\beta} J_{0+}^{\alpha} \lambda_{2}\right)(\tau)-\left({ }^{\beta} J_{0+}^{\alpha} \varphi\right)(\tau)\right]\left[\left({ }^{\beta} J_{0+}^{\alpha} \varphi\right)(\tau)-\left({ }^{\beta} J_{0+}^{\alpha} \lambda_{1}\right)(\tau)\right]} \\
& -\left[{ }^{\beta} J_{0+}^{\alpha}\left(\left(\lambda_{2}(\tau)-\varphi(\tau)\right)\left(\varphi(\tau)-\lambda_{1}(\tau)\right)\right)\right]^{\beta} J_{0+}^{\alpha}(1)-\left[{ }^{\beta} J_{0+}^{\alpha}\left(\left(\lambda_{2}(\tau)-\varphi(\tau)\right)\left(\varphi(\tau)-\lambda_{1}(\tau)\right)\right)\right]^{\beta} J_{0+}^{\alpha}(1) \\
& =\left({ }^{\beta} J_{0+}^{\alpha} \varphi^{2}\right)(\tau)^{\beta} J_{0+}^{\alpha}(1)+\left({ }^{\beta} J_{0+}^{\alpha} \varphi^{2}\right)(\tau)^{\beta} J_{0+}^{\alpha}(1)-2\left({ }^{\beta} J_{0+}^{\alpha} \varphi\right)(\tau)\left({ }^{\beta} J_{0+}^{\alpha} \varphi\right)(\tau)+\left({ }^{\beta} J_{0+}^{\alpha} \lambda_{2}\right)(\tau)\left({ }^{\beta} J_{0+}^{\alpha} \varphi\right)(\tau) \\
& \quad+\left({ }^{\beta} J_{0+}^{\alpha} \varphi\right)(\tau)\left({ }^{\beta} J_{0+}^{\alpha} \lambda_{1}\right)(\tau)-\left({ }^{\beta} J_{0+}^{\alpha} \lambda_{2}\right)(\tau)\left({ }^{\beta} J_{0+}^{\alpha} \lambda_{1}\right)(\tau)+\left({ }^{\beta} J_{0+}^{\alpha} \varphi\right)(\tau)\left({ }^{\beta} J_{0+}^{\alpha} \lambda_{2}\right)(\tau) \\
& \quad+\left({ }^{\beta} J_{0+}^{\alpha} \lambda_{1}\right)(\tau)\left({ }^{\beta} J_{0+}^{\alpha} \varphi\right)(\tau)-\left({ }^{\beta} J_{0+}^{\alpha} \lambda_{1}\right)(\tau)\left({ }^{\beta} J_{0+}^{\alpha} \lambda_{2}\right)(\tau)-\left({ }^{\beta} J_{0+}^{\alpha} \lambda_{2} \varphi\right)(\tau)^{\beta} J_{0+}^{\alpha}(1)+\left({ }^{\beta} J_{0+}^{\alpha} \lambda_{1} \lambda_{2}\right)(\tau)^{\beta} J_{0+}^{\alpha}(1) \\
& \quad-\left({ }^{\beta} J_{0+}^{\alpha} \lambda_{1} \varphi\right)(\tau)^{\beta} J_{0+}^{\alpha}(1)-\left({ }^{\beta} J_{0+}^{\alpha} \lambda_{2} \varphi\right)(\tau)^{\beta} J_{0+}^{\alpha}(1)+\left({ }^{\beta} J_{0+}^{\alpha} \lambda_{1} \lambda_{2}\right)(\tau)^{\beta} J_{0+}^{\alpha}(1)-\left({ }^{\beta} J_{0+}^{\alpha} \lambda_{1} \varphi\right)(\tau)^{\beta} J_{0+}^{\alpha}(1),
\end{aligned}
$$

which leads us to (14).

Remark 2.5. It is not difficult for the reader to verify that Lemma 2.1 contains Theorem 2.2 of [4] and Lemma 7 of [23], as particular cases.

Theorem 2.3. Let $\varphi$ and $\psi$ be two integrable functions on $[0, \infty)$, and let $\gamma_{1}, \gamma_{2}, \eta_{1}$ and $\eta_{2}$ be integrable functions on $[0, \infty)$ satisfying the conditions (4) and (5) for all $\tau>0, \alpha \in \mathbb{R}$ and $\beta \in \mathbb{C}$ with $\operatorname{Re}(\beta)>0$. The following inequality, for the generalized fractional integral operator, is valid:

$$
\left|{ }^{\beta} J_{0+}^{\alpha}(1)\left({ }^{\beta} J_{0+}^{\alpha} \varphi \psi\right)(\tau)-\left({ }^{\beta} J_{0+}^{\alpha} \varphi\right)(\tau)\left({ }^{\beta} J_{0+}^{\alpha} \psi\right)(\tau)\right| \leq \sqrt{R\left(\varphi, \lambda_{1}, \lambda_{2}\right) R\left(\psi, \eta_{1}, \eta_{2}\right)}
$$

where $R(p, q, r)$ is defined as

$$
\begin{aligned}
R(p, q, r)= & \left(\left({ }^{\beta} J_{0+}^{\alpha} r\right)(\tau)-\left({ }^{\beta} J_{0+}^{\alpha} p\right)(\tau)\right)\left(\left({ }^{\beta} J_{0+}^{\alpha} p\right)(\tau)-\left({ }^{\beta} J_{0+}^{\alpha} q\right)(\tau)\right)+{ }^{\beta} J_{0+}^{\alpha}(1)\left({ }^{\beta} J_{0+}^{\alpha} p q\right)(\tau)-\left({ }^{\beta} J_{0+}^{\alpha} q\right)(\tau)\left({ }^{\beta} J_{0+}^{\alpha} p\right)(\tau) \\
& +{ }^{\beta} J_{0+}^{\alpha}(1)\left({ }^{\beta} J_{0+}^{\alpha} r p\right)(\tau)-\left({ }^{\beta} J_{0+}^{\alpha} r\right)(\tau)\left({ }^{\beta} J_{0+}^{\alpha} p\right)(\tau)+\left({ }^{\beta} J_{0+}^{\alpha} q\right)(\tau)\left({ }^{\beta} J_{0+}^{\alpha} r\right)(\tau)-{ }^{\beta} J_{0+}^{\alpha}(1)\left({ }^{\beta} J_{0+}^{\alpha} q r\right)(\tau) .
\end{aligned}
$$

Proof. Suppose that $\varphi$ and $\psi$ are integrable functions on $[0, \infty)$ such that (4) and (8) hold. Let us put

$$
H(\chi, \xi):=(\varphi(\chi)-\varphi(\xi))(\psi(\chi)-\psi(\xi)) ; \quad \chi, \xi \in(0, \tau), \quad \tau>0
$$

Multiply (16) with

$\frac{1}{\Gamma^{2}(\beta)} \cdot \frac{1}{F\left(\mathbb{F}_{+}(\chi, \tau), \beta\right) F(\tau, \alpha)} \cdot \frac{1}{F\left(\mathbb{F}_{+}(\xi, \tau), \beta\right) F(\tau, \alpha)}$,


$\chi, \xi \in(0, \tau)$, and integrate the resulting inequality with respect to $\chi$ and $\xi$ from 0 to $\tau$, we have

$$
\frac{1}{2 \Gamma^{2}(\beta)} \int_{0}^{\tau} \int_{0}^{\tau} \frac{1}{F\left(F_{+}(\chi, \tau), \beta\right) F(\tau, \alpha)} \cdot \frac{1}{F\left(F_{+}(\xi, \tau), \beta\right) F(\tau, \alpha)} H(\chi, \xi) d \chi d \xi={ }^{\beta} J_{0+}^{\alpha}(1)\left({ }^{\beta} J_{0+}^{\alpha} \varphi \psi\right)(\tau)-\left({ }^{\beta} J_{0+}^{\alpha} \varphi\right)(\tau)\left({ }^{\beta} J_{0+}^{\alpha} \psi\right)(\tau) \text {. }
$$

By using Cauchy-Schwarz inequality, we get

$$
\begin{aligned}
& \left({ }^{\beta} J_{0+}^{\alpha}(1)\left({ }^{\beta} J_{0+}^{\alpha} \varphi \psi\right)(\tau)-\left({ }^{\beta} J_{0+}^{\alpha} \varphi\right)(\tau)\left({ }^{\beta} J_{0+}^{\alpha} \psi\right)(\tau)\right)^{2} \\
\leq & \left({ }^{\beta} J_{0+}^{\alpha}(1)\left({ }^{\beta} J_{0+}^{\alpha} \varphi^{2}\right)(\tau)-\left(\left({ }^{\beta} J_{0+}^{\alpha} \varphi\right)(\tau)\right)^{2}\right)\left({ }^{\beta} J_{0+}^{\alpha}(1)\left({ }^{\beta} J_{0+}^{\alpha} \psi^{2}\right)(\tau)-\left(\left({ }^{\beta} J_{0+}^{\alpha} \psi\right)(\tau)\right)^{2}\right) .
\end{aligned}
$$

Now, from the inequalities

$$
\left(\lambda_{2}(\tau)-\varphi(\tau)\right)\left(\varphi(\tau)-\lambda_{1}(\tau)\right) \geq 0
$$

and

$$
\left(\eta_{2}(\tau)-\psi(\tau)\right)\left(\psi(\tau)-\eta_{1}(\tau)\right) \geq 0
$$

for $\tau \in[0, \infty)$, we obtain

$$
{ }^{\beta} J_{0+}^{\alpha}(1)^{\beta} J_{0+}^{\alpha}\left(\lambda_{2}(\tau)-\varphi(\tau)\right)\left(\varphi(\tau)-\lambda_{1}(\tau)\right) \geq 0
$$

and

$$
{ }^{\beta} J_{0+}^{\alpha}(1)^{\beta} J_{0+}^{\alpha}\left(\eta_{2}(\tau)-\psi(\tau)\right)\left(\psi(\tau)-\eta_{1}(\tau)\right) \geq 0
$$

By using Lemma 2.1, we have

$$
\begin{aligned}
\left({ }^{\beta} J_{0+}^{\alpha}(1)\left({ }^{\beta} J_{0+}^{\alpha} \varphi^{2}\right)(\tau)-\left(\left({ }^{\beta} J_{0+}^{\alpha} \varphi\right)(\tau)\right)^{2}\right) \leq & \left(\left({ }^{\beta} J_{0+}^{\alpha} \lambda_{2}\right)(\tau)-\left({ }^{\beta} J_{0+}^{\alpha} \varphi\right)(\tau)\right)\left(\left({ }^{\beta} J_{0+}^{\alpha} \varphi\right)(\tau)-\left({ }^{\beta} J_{0+}^{\alpha} \lambda_{1}\right)(\tau)\right) \\
& +{ }^{\beta} J_{0+}^{\alpha}(1)\left({ }^{\beta} J_{0+}^{\alpha} \lambda_{1} \varphi\right)(\tau)-\left({ }^{\beta} J_{0+}^{\alpha} \lambda_{1}\right)(\tau)\left({ }^{\beta} J_{0+}^{\alpha} \varphi\right)(\tau) \\
& +{ }^{\beta} J_{0+}^{\alpha}(1)\left({ }^{\beta} J_{0+}^{\alpha} \lambda_{2} \varphi\right)(\tau)-\left({ }^{\beta} J_{0+}^{\alpha} \lambda_{2}\right)(\tau)\left({ }^{\beta} J_{0+}^{\alpha} \varphi\right)(\tau) \\
& +\left({ }^{\beta} J_{0+}^{\alpha} \lambda_{1}\right)(\tau)\left({ }^{\beta} J_{0+}^{\alpha} \lambda_{2}\right)(\tau)-{ }^{\beta} J_{0+}^{\alpha}(1)\left({ }^{\beta} J_{0+}^{\alpha} \lambda_{1} \lambda_{2}\right)(\tau) \\
= & R\left(\varphi, \lambda_{1}, \lambda_{2}\right)
\end{aligned}
$$

and

$$
\begin{aligned}
\left({ }^{\beta} J_{0+}^{\alpha}(1)\left({ }^{\beta} J_{0+}^{\alpha} \psi^{2}\right)(\tau)-\left(\left({ }^{\beta} J_{0+}^{\alpha} \psi\right)(\tau)\right)^{2}\right) \leq & \left(\left({ }^{\beta} J_{0+}^{\alpha} \eta_{2}\right)(\tau)-\left({ }^{\beta} J_{0+}^{\alpha} \psi\right)(\tau)\right)\left(\left({ }^{\beta} J_{0+}^{\alpha} \psi\right)(\tau)-\left({ }^{\beta} J_{0+}^{\alpha} \eta_{1}\right)(\tau)\right) \\
& +{ }^{\beta} J_{0+}^{\alpha}(1)\left({ }^{\beta} J_{0+}^{\alpha} \eta_{1} \psi\right)(\tau)-\left({ }^{\beta} J_{0+}^{\alpha} \eta_{1}\right)(\tau)\left({ }^{\beta} J_{0+}^{\alpha} \psi\right)(\tau) \\
& +{ }^{\beta} J_{0+}^{\alpha}(1)\left({ }^{\beta} J_{0+}^{\alpha} \eta_{2} \psi\right)(\tau)-\left({ }^{\beta} J_{0+}^{\alpha} \eta_{2}\right)(\tau)\left({ }^{\beta} J_{0+}^{\alpha} \psi\right)(\tau) \\
& +\left({ }^{\beta} J_{0+}^{\alpha} \eta_{1}\right)(\tau)\left({ }^{\beta} J_{0+}^{\alpha} \eta_{2}\right)(\tau)-{ }^{\beta} J_{0+}^{\alpha}(1)\left({ }^{\beta} J_{0+}^{\alpha} \eta_{1} \eta_{2}\right)(\tau) \\
= & R\left(\psi, \eta_{1}, \eta_{2}\right) .
\end{aligned}
$$

By using (17), (18) and (19), we arrive at (15).

Remark 2.6. It is not difficult to see that Theorem 2.3 of [4], Theorem 9 of [23] and Theorem 3.1 of [6] are the special cases of Theorem 2.3.

Remark 2.7. The results obtained in [12], in the framework of Riemann-Liouville fractional integral of a function $f(x)$ with respect to another function $h(x)$, can also be derived from those presented in this paper.

\section{Conclusions}

In this work, we have obtained several integral inequalities, of the Grüss type, from which various known results are obtained as particular cases. We note that the generalized integral operator given in Definition 1.2 contains several wellknown fractional operators. Some examples are given below.

- If we take $F(\tau, \alpha)=\tau^{-\alpha}$ with $\alpha=1$, we get the $k$-Riemann-Liouville fractional integral of Mubeen and Habibullah [16]. The right sided operator, in this case, is given as

$$
\left(\frac{\beta}{k} J_{F, a+}^{\alpha} \varphi\right)(\chi)=\frac{1}{k \Gamma_{k}(\beta)} \int_{a}^{\chi} \frac{\varphi(\tau) d \tau}{(\chi-\tau)^{1-\frac{\beta}{k}}}
$$

and similarly the left sided integral can be written. 
- The choice $F(\tau, \alpha)=\tau^{-\alpha}$ gives us the Katugampola fractional integral [13] (the notation is changed).

- The case $F=\tau^{\alpha}$ with $\alpha=1$ corresponds to the right sided Hadamard fractional integral [11].

- An integral operator with non-singular kernel can also be obtained from Definition 1.2. Let us consider $F(\tau, \alpha)=$ $\exp \left[\frac{1-\alpha}{\alpha} \tau\right]$. If $\alpha=1$ then we have $F=1$. In this case,

$$
F\left(\mathbb{F}_{+}(\chi, \tau), \beta\right)=\exp \left[\frac{1-\beta}{\beta}(\chi-\tau)\right]
$$

which is a slight modification of the operator defined by Kirane and Toberek [1].

This shows the possibilities of future work by extending various known results in the generalized context of Definition 1.2.

\section{References}

[1] B. Ahmad, A. Alsaedi, M. Kirane, B. T. Toberek, Hermite-Hadamard, Hermite-Hadamard-Fejer, Dragomir-Agarwal and Pachpatte type inequalities for convex functions via new integrals, J. Comput. Appl. Math. 353 (2019) 120-129.

[2] M. W. Alomari, A companion of Grüss type inequality for Riemann-Stieltjes integral and applications, Mat. Vesnik 66 (2014) $202-212$.

[3] D. Baleanu, S. D. Purohit, F. Uçar, On Grüss type integral inequality involving the Saigo's fractional integral operators, J. Comput. Anal. Appl. 19 (2015) 480-489.

[4] B. Celik, E. Set, A. O. Akdemir, Mixed Conformable Fractional Grüss-Type Inequalities, 2nd International Conference on Life and Engineering Sciences (ICOLES 2019), Istanbul, Turkey, 2019.

[5] P. Cerone, S. S. Dragomir, A refinement of the Grüss inequality and applications, Tamkang J. Math. 38 (2007) 37-49.

[6] Z. Dahmani, L. Tabharit, S. Taf, New generalisations of Grüss inequality using Riemann-Liouville fractional integrals, Bull. Math. Anal. Appl. 2 (2010) 93-99.

[7] S. S. Dragomir, Some integral inequalities of Grüss type, Indian J. Pure Appl. Math. 31 (2000) 397-415.

[8] S. S. Dragomir, A companion of the Grüss inequality and applications, Appl. Math. Lett. 17 (2004) 429-435.

[9] G. Grüss, Uber das Maximum des absoluten Betrages von $\frac{1}{b-a} \int_{a}^{b} f(x) g(x) d x-\frac{1}{(b-a)^{2}} \int_{a}^{b} f(x) d x \int_{a}^{b} g(x) d x$, Math. Z. 39 (1935) $215-226$.

[10] P. M. Guzmán, J. E. Nápoles, Y. Gasimov, Integral inequalities within the framework of generalized fractional integrals, Submitted.

[11] J. Hadamard, Etude sur les propriétés des fonctions entiéres et en particulier d'une fonction considérée par Riemann, J. Math. Pures Appl. 9 (1893) $171-216$.

[12] E. Kacar, Z. Kacar, H. Yildirim, integral inequalities for Riemann-Liouville fractional integrals of a function with respect to another function, Iran. J. Math. Sci. Inform. 13 (2018) 1-13.

[13] U. N. Katugampola, New approach generalized fractional integral, Appl. Math. Comput. 218 (2011) 860-865.

[14] Z. Liu, Notes on a Grüss type inequality and its application, Vietnam J. Math. 35 (2007) 121-127.

[15] A. M. Mercer, An improvement of the Grüss inequality, J. Inequal. Pure Appl. Math. 6 (2005) Art\# 93.

[16] S. Mubeen, G. M. Habibullah, $k$-fractional integrals and applications. Int. J. Contemp. Math. Sci. 7 (2012) 89-94.

[17] A. M. Mercer, P. R. Mercer, New proofs of the Grüss inequality, Aust. J. Math. Anal. Appl. 1 (2004) Art\# 12.

[18] Y. Miao, F. Han, J. Mu, A new Ostrowski-Grüss type inequality, Kragujevac J. Math. 37 (2013) 307-317.

[19] D. S. Mitrinovic, J. E. Pecaric, A. M. Fink, Inequalities for Functions and Their Integrals and Derivatives, Kluwer Academic, Dordrecht, 1994.

[20] B. G. Pachpatte, On Grüss type integral inequalities, J. Inequal. Pure Appl. Math. 3 (2002) Art\# 27.

[21] M. Z. Sarikaya, H. Budak, An inequality of Grüss like via variant of Pompeiu's mean value theorem, Konuralp J. Math. 3 (2015) 29-35.

[22] E. Set, M. Tomar, M. Sarikaya, On generalized Grüss type inequalities for $k$-fractional integrals, Appl. Math. Comput. 269 (2015) 29-34.

[23] J. Tariboon, S. K. Ntouyas, W. Sudsutad, Some new Riemann-Liouville fractional integral inequalities, Int. J. Math. Math. Sci. 2014 (2014) Art\# 869434.

[24] J. Vanterler, D. S. Oliveira, E. Capelas de Oliveira, Grüss-type inequalities by means of generalized fractional integrals, Bull. Braz. Math. Soc. (N.S.) 50 (2019) 1029-1047. 